\title{
Crystal structure of 2-((E)-(6-methylpyridin-2-ylimino)methyl)-4- methylphenol, $\mathrm{C}_{14} \mathrm{H}_{14} \mathrm{~N}_{2} \mathrm{O}$
}

\author{
Fang-Fang Dang ${ }^{*}$, I Dang-Yang Zhao ${ }^{\mathrm{II}}$ and Hui Guo ${ }^{\mathrm{III}}$ \\ I School of Science, Xi'an University of Architecture and Technology, Xi'an 710055, Shaanxi Province, P. R. China \\ ${ }^{\text {II }}$ Luohe Environmental Sanitation Management Agency, Luohe 462000, Henan Province, P. R. China

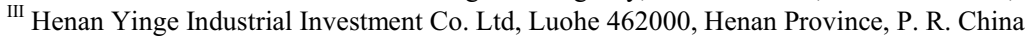

Received October 25, 2013, accepted January 28, 2014, available online February 28, 2014, CCDC no. 1267/4056

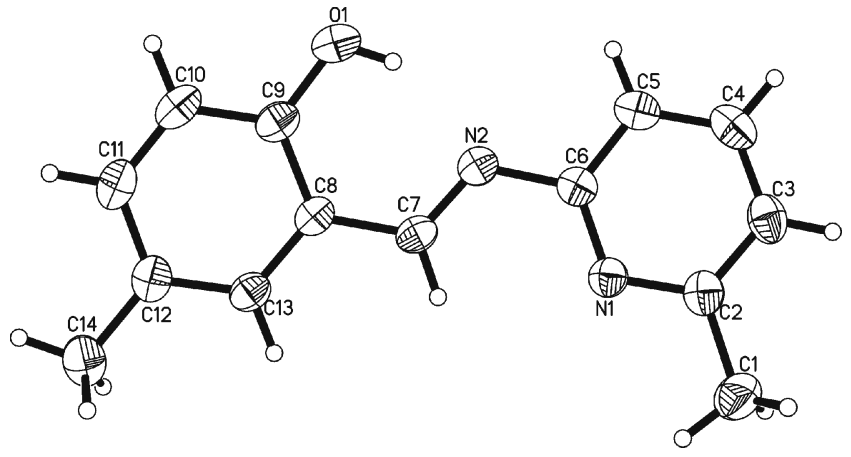

\section{Abstract}

$\mathrm{C}_{14} \mathrm{H}_{14} \mathrm{~N}_{2} \mathrm{O}$, monoclinic, $P 2_{1} / n$ (no. 14), $a=16.0418(9) \AA$, $b=4.6119(2) \AA, c=17.0111(9) \AA, \beta=106.585(6)^{\circ}$,

$V=1206.2 \AA^{3}, Z=4, R_{\mathrm{gt}}(F)=0.0660, w R_{\mathrm{ref}}\left(F^{2}\right)=0.1994$,

$T=293 \mathrm{~K}$.

Table 1. Data collection and handling.

$\begin{array}{ll}\text { Crystal: } & \text { yellow blocks, size } 0.16 \times 0.25 \times 0.42 \mathrm{~mm} \\ \text { Wavelength: } & \text { Mo } K_{\alpha} \text { radiation }(0.71073 \AA) \\ \mu: & 0.80 \mathrm{~cm}^{-1} \\ \text { Diffractometer, scan mode: } & \text { Rigaku R-AXIS RAPID, } \omega \\ 2 \theta_{\text {max }}: & 50^{\circ} \\ N(h k l)_{\text {measured }}, N(h k l)_{\text {unique }}: & 5753,2118 \\ \text { Criterion for } I_{\text {obs }}, N(h k l)_{\text {gt }}: & I_{\text {obs }}>2 \sigma\left(I_{\text {obs }}\right), 1574 \\ N(\text { param })_{\text {refined }}: & 154 \\ \text { Programs: } & \text { SHELX }[10]\end{array}$

\section{Source of material}

2-Hydroxybenzaldehyde (2.4 g, $20 \mathrm{mmol})$ and 6-methylpyridin2-amine (2.2 g, $20 \mathrm{mmol}$ ) were dissolved in ethanol respectively. Then these were put together and the mixture was refluxed for 30 minutes. Yellow powder precipitates during the cooling to room temperature. After evaporation, a crude product was recrystallized twice from carbinol to give a pure yellow product. Elemental Analysis calcd. for $\mathrm{C}_{14} \mathrm{H}_{14} \mathrm{~N}_{2} \mathrm{O}$ : C, 74.31; H, 6.24; N, 12.38; Found: C, 74.27; H, 6.28; N, 12.37 .

\section{Discussion}

The Schiff base compounds and their derivatives have been playing an important role in the development of coordination chemistry $[1,2]$ because these compounds can accommodate one, two or more metal centers and form homo- and heteronuclear metal complexes with interesting properties [3-6], such as excellent catalytic activities for epoxidation, aziridination, etc. [7], models of reaction centers of metalloenzymes [8], and nonlinear optical materials [9]. Here we designed and synthesized a new Schiff base named 2-((E)-(6-methylpyridin-2-ylimino)methyl)-4methylphenol. The crystal structure of the title compound is only built up by the $\mathrm{C}_{14} \mathrm{H}_{14} \mathrm{~N}_{2} \mathrm{O}$ molecules, in which all bond lengths are in normal ranges. The $\mathrm{C} 7=\mathrm{N} 2$ bond length is 1.281(4) $\AA$ whereas the C6-N2 bond length is found to be 1.418(3) $\AA$. The $\mathrm{C} 6, \mathrm{~N} 2$ and $\mathrm{C} 7$ atoms form a bond angle of $120.5(2)^{\circ}$. The molecular conformation is stabilized by an intramolecular $\mathrm{O}-\mathrm{H} \cdots \mathrm{N}$ hydrogen bond (O1-H1D $\cdots \mathrm{N} 2)$.

Table 2. Atomic coordinates and displacement parameters (in $\AA^{2}$ ).

\begin{tabular}{llllrl}
\hline Atom & Site & $x$ & $y$ & \multicolumn{1}{l}{$z$} & $U_{\text {iso }}$ \\
\hline $\mathrm{H}(7 \mathrm{~A})$ & $4 e$ & 0.9080 & 1.0142 & 0.2277 & 0.059 \\
$\mathrm{H}(13 \mathrm{~A})$ & $4 e$ & 0.9627 & 1.3680 & 0.3370 & 0.063 \\
$\mathrm{H}(10 \mathrm{~A})$ & $4 e$ & 1.2117 & 1.5135 & 0.2576 & 0.079 \\
$\mathrm{H}(3 \mathrm{~A})$ & $4 e$ & 0.7353 & 0.1859 & -0.0018 & 0.077 \\
$\mathrm{H}(5 \mathrm{~A})$ & $4 e$ & 0.9425 & 0.6757 & 0.0052 & 0.073 \\
$\mathrm{H}(11 \mathrm{~A})$ & $4 e$ & 1.1930 & 1.7517 & 0.3696 & 0.077 \\
$\mathrm{H}(4 \mathrm{~A})$ & $4 e$ & 0.8385 & 0.3425 & -0.0633 & 0.082 \\
$\mathrm{H}(14 \mathrm{~A})$ & $4 e$ & 1.1136 & 1.8654 & 0.4625 & 0.113 \\
$\mathrm{H}(14 \mathrm{~B})$ & $4 e$ & 1.0581 & 1.6065 & 0.4800 & 0.113 \\
$\mathrm{H}(14 \mathrm{C})$ & $4 e$ & 1.0125 & 1.8631 & 0.4236 & 0.113 \\
$\mathrm{H}(1 \mathrm{D})$ & $4 e$ & 1.0702 & 1.0554 & 0.1411 & 0.110 \\
$\mathrm{H}(1 \mathrm{~A})$ & $4 e$ & 0.7213 & 0.4405 & 0.1932 & 0.124 \\
$\mathrm{H}(1 \mathrm{~B})$ & $4 e$ & 0.7161 & 0.1346 & 0.1519 & 0.124 \\
$\mathrm{H}(1 \mathrm{C})$ & $4 e$ & 0.6536 & 0.3836 & 0.1078 & 0.124 \\
\hline
\end{tabular}

\footnotetext{
* Correspondence author (e-mail: dff516@163.com)
} 
Table 3. Atomic coordinates and displacement parameters (in $\AA^{2}$ ).

\begin{tabular}{|c|c|c|c|c|c|c|c|c|c|c|}
\hline Atom & Site & $x$ & $y$ & $z$ & $U_{11}$ & $U_{22}$ & $U_{33}$ & $U_{12}$ & $U_{13}$ & $U_{23}$ \\
\hline $\mathrm{N}(2)$ & $4 e$ & $0.9621(1)$ & $0.9117(5)$ & $0.1448(1)$ & $0.048(1)$ & $0.052(1)$ & $0.054(1)$ & $0.003(1)$ & $0.014(1)$ & $0.005(1)$ \\
\hline$C(7)$ & $4 e$ & $0.9562(2)$ & $1.0463(6)$ & $0.2088(2)$ & $0.043(1)$ & $0.050(2)$ & $0.056(2)$ & $0.004(1)$ & $0.016(1)$ & $0.009(1)$ \\
\hline $\mathrm{C}(8)$ & $4 e$ & $1.0226(2)$ & $1.2468(6)$ & $0.2526(2)$ & $0.040(1)$ & $0.045(1)$ & $0.052(1)$ & $0.003(1)$ & $0.009(1)$ & $0.013(1)$ \\
\hline $\mathrm{N}(1)$ & $4 e$ & $0.8366(1)$ & $0.6280(5)$ & $0.1400(1)$ & $0.046(1)$ & $0.055(1)$ & $0.050(1)$ & $0.002(1)$ & $0.0106(9)$ & $0.001(1)$ \\
\hline$C(13)$ & $4 e$ & $1.0129(2)$ & $1.3973(6)$ & $0.3212(2)$ & $0.048(1)$ & $0.051(2)$ & $0.058(2)$ & $0.000(1)$ & $0.015(1)$ & $0.010(1)$ \\
\hline$C(6)$ & $4 e$ & $0.8967(2)$ & $0.7123(6)$ & $0.1040(2)$ & $0.046(1)$ & $0.049(2)$ & $0.048(1)$ & $0.010(1)$ & $0.009(1)$ & $0.006(1)$ \\
\hline $\mathrm{C}(3)$ & $4 e$ & $0.7768(2)$ & $0.3221(7)$ & $0.0242(2)$ & $0.061(2)$ & $0.062(2)$ & $0.061(2)$ & $0.004(2)$ & $0.001(1)$ & $-0.010(2)$ \\
\hline $\mathrm{C}(12)$ & $4 e$ & $1.0751(2)$ & $1.5875(6)$ & $0.3660(2)$ & $0.057(2)$ & $0.048(2)$ & $0.056(2)$ & $0.001(1)$ & $0.004(1)$ & $0.011(1)$ \\
\hline $\mathrm{C}(9)$ & $4 e$ & $1.0984(2)$ & $1.2953(6)$ & $0.2283(2)$ & $0.050(2)$ & $0.053(2)$ & $0.062(2)$ & $0.002(1)$ & $0.017(1)$ & $0.014(1)$ \\
\hline$C(5)$ & $4 e$ & $0.8998(2)$ & $0.6112(7)$ & $0.0282(2)$ & $0.062(2)$ & $0.067(2)$ & $0.056(2)$ & $0.010(2)$ & $0.022(1)$ & $0.003(2)$ \\
\hline $\mathrm{C}(2)$ & $4 e$ & $0.7767(2)$ & $0.4337(6)$ & $0.0997(2)$ & $0.049(2)$ & $0.056(2)$ & $0.055(2)$ & $0.005(1)$ & $0.004(1)$ & $0.002(1)$ \\
\hline $\mathrm{C}(11)$ & $4 e$ & $1.1498(2)$ & $1.6261(7)$ & $0.3404(2)$ & $0.054(2)$ & $0.059(2)$ & $0.070(2)$ & $-0.009(1)$ & $0.003(1)$ & $0.011(2)$ \\
\hline $\mathrm{C}(4)$ & $4 e$ & $0.8383(2)$ & $0.4134(7)$ & $-0.0122(2)$ & $0.075(2)$ & $0.076(2)$ & $0.052(2)$ & $0.017(2)$ & $0.014(2)$ & $-0.009(2)$ \\
\hline $\mathrm{C}(14)$ & $4 e$ & $1.0638(2)$ & $1.7448(7)$ & $0.4397(2)$ & $0.085(2)$ & $0.068(2)$ & $0.067(2)$ & $-0.006(2)$ & $0.011(2)$ & $-0.001(2)$ \\
\hline $\mathrm{C}(1)$ & $4 e$ & $0.7110(2)$ & $0.3397(9)$ & $0.1419(2)$ & $0.065(2)$ & $0.101(3)$ & $0.082(2)$ & $-0.021(2)$ & $0.021(2)$ & $-0.005(2)$ \\
\hline
\end{tabular}

Acknowledgments. This work was supported by the Education Department of Shanxi province a special research project 09JK512.

\section{References}

1. Boghaei, D.-M.; Bezaatpour, A.; Behzad, M.: Synthesis, characterization and catalytic activity of novel monomeric and polymeric vanadyl Schiff base complexes. J. Mol. Catal. A245 (2006) 12-16.

2. Dong, W.-K.; Duan, J.-G.; Guan, Y.-H.; Shi, J.-Y.; Zhao, C.-Y.: Synthesis, crystal structure and spectroscopic behaviors of $\mathrm{Co}(\mathrm{II})$ and $\mathrm{Cu}(\mathrm{II})$ complexes with Salen-type bisoxime ligands. Inorg. Chim. Acta 362 (2009) 1129-1134.

3. Dong, W.-K.; He, X.-N.; Yan, H.-B.; Lv, Z.-W.; Chen, X.; Zhao, C.-Y.; Tang, X.-L.: Synthesis, structural characterization and solvent effect of copper(II) complexes with a variational multidentate Salen-type ligand with bisoxime groups. Polyhedron 28 (2009) 1419-1428.

4. Dong, W.-K.; Li, L.; Li, C.-F.; Xu, L.; Duan, J.-G.: Synthesis, structure and spectroscopic behaviors of a five- and six-coordinated tri-cobalt(II) cluster $\left[(\mathrm{CoL})_{2}(\mathrm{OAc})_{2} \mathrm{Co}\right] \cdot 2 \mathrm{C}_{2} \mathrm{H}_{5} \mathrm{OH}$. Spectrochimica Acta A71 (2008) 650-654.
5. Dong, W.-K.; Sun, Y.-X.; He, X.-N.; Tong, J.-F.; Wu, J.-C.: Trinuclear and mononuclear copper(II) complexes incorporating tetradentate 2,2'[1,1'-(ethylenedioxydinitrilo)diethylidyne]diphenol ligand: Syntheses, crystal structures, spectral and thermal behaviors. Spectrochimica Acta A76 (2010) 476-483.

6. Dong, W.-K.; Sun, Y.-X.; Zhang, Y.-P.; Li, L.; He, X.-N.; Tang, X.-L.: Synthesis, crystal structure, and properties of supramolecular CuII, ZnII, and CdII complexes with Salen-type bisoxime ligands. Inorg. Chim. Acta 362 (2009) 117-124.

7. Dong, W.-K.; Sun, Y.-X.; Zhao, C.-Y.; Dong, X.-Y.; Xu, L.: Synthesis, structure and properties of supramolecular MnII, CoII, NiII and ZnII complexes containing Salen-type bisoxime ligands. Polyhedron 29 (2010) 2087-2097.

8. Lacroix, P. G.: Second-order optical nonlinearities in coordination chemistry: The case of bis(salicylaldiminato)metal Schiff base complexes. Eur. J. Inorg. Chem. (2001) 339-348.

9. Summers, M. F.; Marzilli, L. G.; Bresciani-Pahor, N.; Randaccio, L.: Unusual bond lengths, conformations, and ligand exchange rates in B12 models with the bis(salicylidene)- $o$-phenylenediamine equatorial ligand. J. Am. Chem. Soc. 106 (1984) 4478-4485.

10. Sheldrick, G. M.: A short history of SHELX. Acta Crystallogr. A64 (2008) 112-122. 